\title{
Módulos de entrenamiento para la medición y adquisición de datos (Módulo de visión)
}

\author{
I. Mejia, J. Gutiérrez y V. Moncada* \\ Facultad de Electrotecnia y Computación, Universidad Nacional de Ingeniería (UNI) \\ PO Box 5595, Managua, Nicaragua \\ e-mail: vimoncada@uni.edu.ni
}

(recibido/received: 20-Septiembre-2009; aceptado/accepted: 21-noviembre-2009)

\begin{abstract}
RESUMEN
En este artículo se presenta el proyecto de diseño e implementación de una herramienta de laboratorio, que pretende contribuir a solucionar el déficit existente de medios de entrenamiento para los estudiantes de la carrera de Ingeniería Electrónica de la Universidad Nacional de Ingeniería. El proyecto está enmarcado dentro del área de automatización industrial, específicamente en la adquisición y procesamiento de imágenes. El sistema de Visión Artificial implementado tiene capacidad de permitir la realización de diferentes prácticas en el área de adquisición y procesamiento de imágenes. Entre las aplicaciones que este sistema ofrece se destacan: Detección de fallas en la fabricación de diferentes productos tales como: Fusibles, Integrados con encapsulado HSIP, Objetos circulares, etc; Lectura de códigos de barras; Reconocimiento óptico de caracteres; Supervisión óptica de indicadores digitales (LCD) y analógicos. Los componentes de hardware cumplen con los estándares de la norma IEEE 1394 (Sensor y Hardware de Adquisición) y el software de procesamiento se implementó utilizando la plataforma LabVIEW. Dentro de los componentes del hardware, se diseñaron diferentes escenarios que incluyeron la posición de la cámara y la cantidad de iluminación en función del objeto a inspeccionar y que a su vez garantizó la adquisición adecuada de los datos, lo que permitió evaluar y contrastar sus usos y aplicaciones. Por su parte el software, ejemplifica los diferentes métodos y algoritmos de procesamiento de imágenes, recreando aplicaciones comunes en la industria nicaragüense. Para asegurar el aprovechamiento y uso adecuado del sistema se incluyó la documentación necesaria para conocer el sistema, lo cual es necesario para su correcto uso y mantenimiento.
\end{abstract}

Palabras claves: maquinas de visión, procesamiento de imágenes, control de calidad, adquisición de datos.

\begin{abstract}
The Project proposes a design for the implementation of a laboratory tool which aims to contribute toward a solution for the current defecit in methods of student training in electrical engineering in the National University of Engineering. As well as in the area of industrial automatization, specifically in the acquisition and processing of images. The system of artificial vision to be implemented has the capacity to allow for different practical studies in the areas of acquisition and image processing. Included in the application are the following: the detection of manufacturing faults in different products, such as, electrical fuses integrated within HSIP capping, circular objects etc; Information on bar codes; Visual character recognition; Visual oversight of analogical, digital indicators (LCD). The hardware components meet the regulation standards IEEE 1394 (Sensor and Acquisition Hardware) and the processing software is implemented using the platform of LabVIEW. Different scenarios have been incorporated into the hardware component. These include, camera position, amount of illumination in relation to the object under scrutiny and guaranteed acquisition of data with which to evaluate and compare use and application. The software exemplifies the different methods and algorithms of image processing, recreating applications which are typically found in Nicaraguan Industry. To ensure the greatest advantage and adequate use, the necessary documentation regarding use and maintainance of the system is included.
\end{abstract}

Keywords. Viewing machines, image processing, quality control, data acquisition.

\footnotetext{
${ }^{*}$ Autor para la correspondencia
} 


\section{INTRODUCCIÓN}

Los sistemas de visión de máquinas comenzaron a aparecer a principios de la década de los años 50 con fines investigativos y actualmente están siendo utilizados ampliamente en la industria para la supervisión automática de líneas de producción, permitiendo el aumento de la productividad sin perder el control de calidad de los productos, lo que permitirá aumentar los estándares de calidad internacionales.

El objetivo general de un sistema de visión artificial es obtener una descripción de una escena, analizando regiones de interés específicas de las que se extraerán rasgos de interés para su correcto análisis e interpretación. El sistema de visión está conformado por diversos componentes, cada uno de ellos cruciales para el análisis y procesamiento de imágenes:

1. Cámara (sensor), que capta las variaciones de luz en los objetos.

2. Una tarjeta de adquisición de imágenes (Frame Grabber), que es la interfaz entre el sensor y la unidad de procesamiento que es una computadora.

3. Software de procesamiento de imágenes, NI Visión para LabVIEW.

4. Sistema de iluminación, el cual es un componente que determina la calidad de la imagen adquirida para su posterior procesamiento.

\section{METODOLOGÍA}

Con el fin de optimizar el empleo de una sola cámara para la inspección de diferentes características en distintos objetos, se requirió de un sistema mecánico que permitió la ubicación de la misma en diferentes posiciones según el tipo de objeto que se estaba analizando. De esta manera se amplió el rango de posibilidades de aplicación que pueden ser llevadas a cabo con este sistema.

La estructura proporciona cuatro distintos movimientos permitiendo localizar a la cámara en casi todos los ángulos de un objeto que requiere diversas tomas como es el caso de las botellas, en las se hace una inspección de pico desde la parte superior y un análisis en los costados para detectar suciedad o fisuras en ellas.

Los movimientos se lograron a través de tres motores sincrónicos ubicados en puntos esenciales los que permitieron dar un movimiento de 180 grados al brazo sobre el cual descansa la cámara, un movimiento desde arriba hasta abajo para pasar de una inspección superior hacia una lateral, y un movimiento que coloca la cámara en dirección vertical u horizontal.

Los objetos fueron colocados sobre una mesa metálica que gira de forma constante para simular la supervisión continua del flujo de productos en una línea de producción.

Los movimientos de los motores se controlan a través de un PLC (Controlador Lógico Programable), Allen Bradley MicroLogic 1000, el cual posee un programa que proporciona la seguridad necesaria para evitar movimientos indebidos que vayan a ocasionar fallas o, en el caso extremo, daños al sistema.

El sistema de iluminación está formado por cuatro alógenos AR11 de 50 Watts, 12 Voltios con una protección anti-deslumbramiento. La intensidad de luz de los alógenos es ajustada por un dimmer. El software de visión contempla parámetros que permiten fijar la intensidad de luz en dependencia del objeto que se esté identificando, permitiendo controlar por medio del puerto paralelo al dimmer con un motor paso a paso.

El software para el procesamiento de imágenes se diseñó bajo la plataforma labVIEW ver. 8.0 utilizando una tarjeta de adquisición de la National Instruments modelo NI PCI-8252, la que proporciona tres puertos IEEE $1394^{\mathrm{a}}$, una velocidad de trasferencia superior a $400 \mathrm{Mb} / \mathrm{s}$ y una cámara Bassler modelo A601f, monocromática que proporciona una resolución de $640 \times 480$, interfaz IEEE 1394.

Para demostrar la utilidad del sistema de visión artificial se desarrollaron las siguientes aplicaciones:

\section{Inspección de fusibles (ver figura 1):}

Este programa permitió verificar el estado de los fusibles de manera automática a través la técnica Pattern Matching. Con esta técnica y mediante el uso de una plantilla de referencia se compara la intensidad de los pixeles de la imagen adquirida con la intensidad de los pixeles de la plantilla, el sistema de coordenadas es actualizado para localizar el objeto en cualquier posición de la imagen. 


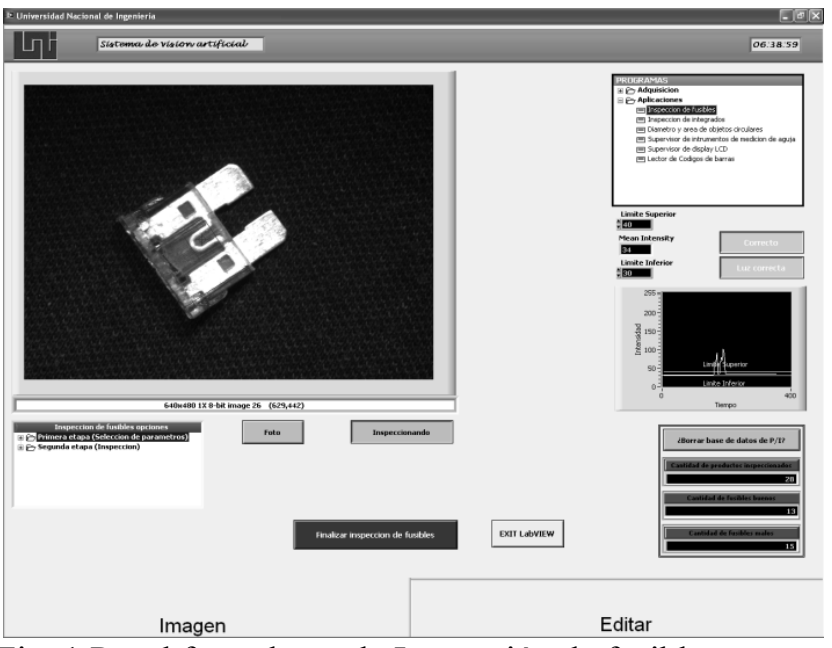

Fig. 1 Panel frontal para la Inspección de fusibles

2. Lectura de código de barras (ver figura 2).

Este programa permite tener un control de los productos, mediante la lectura de su código de barras independientemente de los tipos que esté utilizando la industria, entre los cuales se pueden mencionar el código de barras en una dimensión, en dos dimensiones (matriz de datos) y el PDF 147. Además el programa permite visualizar, agregar, remplazar o borrar productos almacenados en una base de datos.

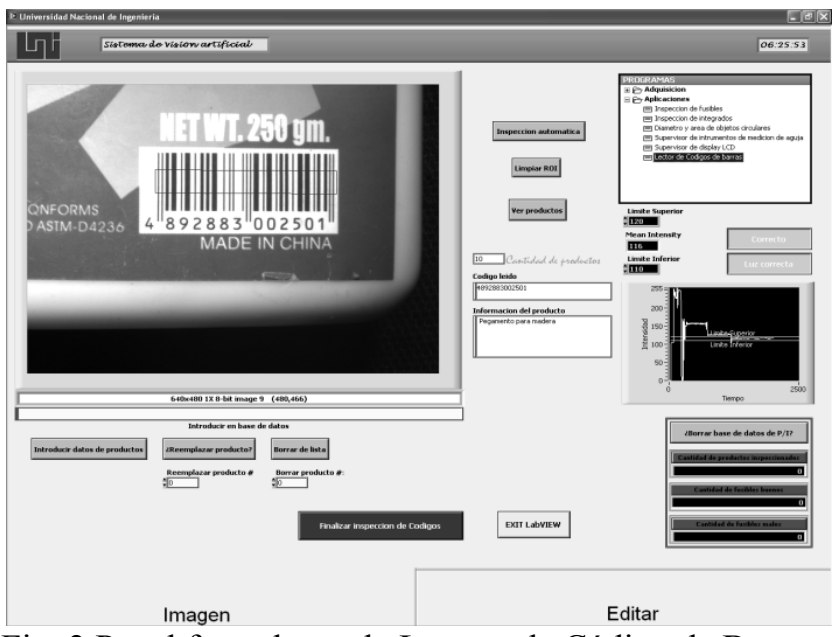

Fig. 2 Panel frontal para la Lectura de Código de Barras.

3. Supervisión de indicador analógico (ver figura 3).

Este programa permite tener una supervisión automática de un indicador analógico a través de la herramienta Detección de Bordes. En la figura 3 se utiliza un multímetro analógico.

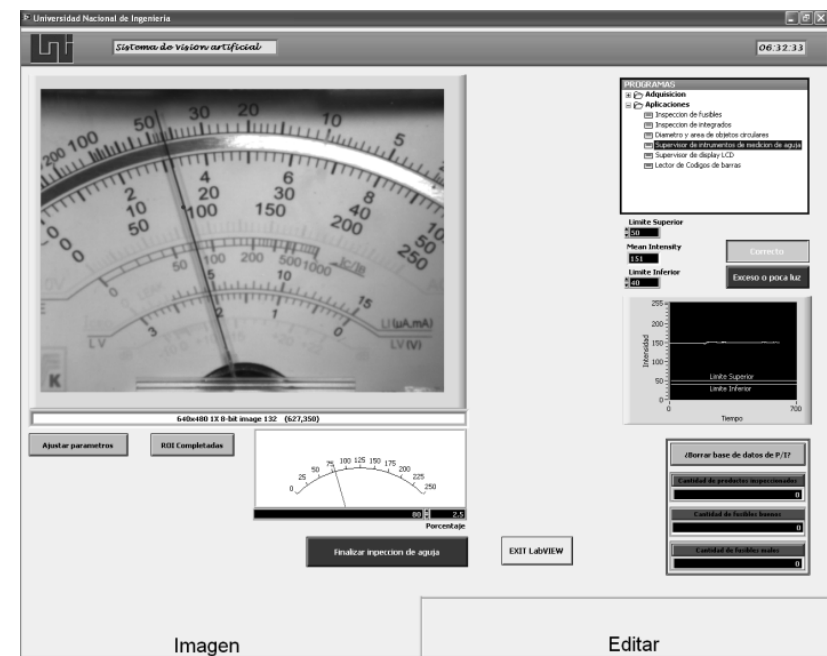

Fig. 3 Panel frontal para la Supervisión de un Indicador Analógico

4. Supervisor de indicador digital LCD (ver figura 4). Este programa permite tener la supervisión de un indicador digital LCD.

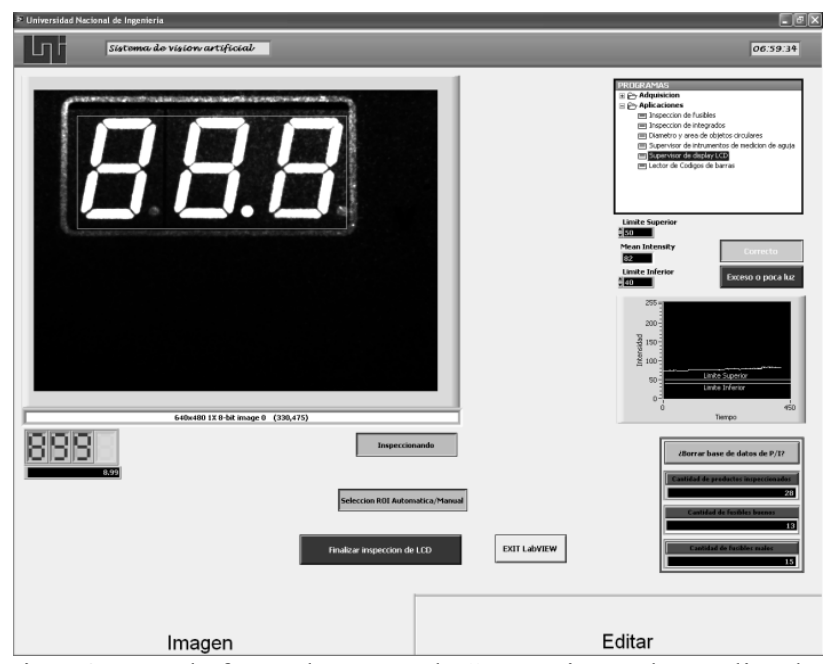

Fig. 4 Panel frontal para el Supervisor de Indicador digital LCD.

\section{Análisis de objetos circulares (ver figura 5).}

Este programa controla los errores de tamaño en objetos circulares calculando el diámetro y área de los mismos. Esto se logra a través de una calibración previa de la imagen convirtiendo las medidas en pixeles a valores de ingeniería. Una vez calibrada la imagen se procede a segmentarla para separar el objeto del fondo y calcular sus medidas. 


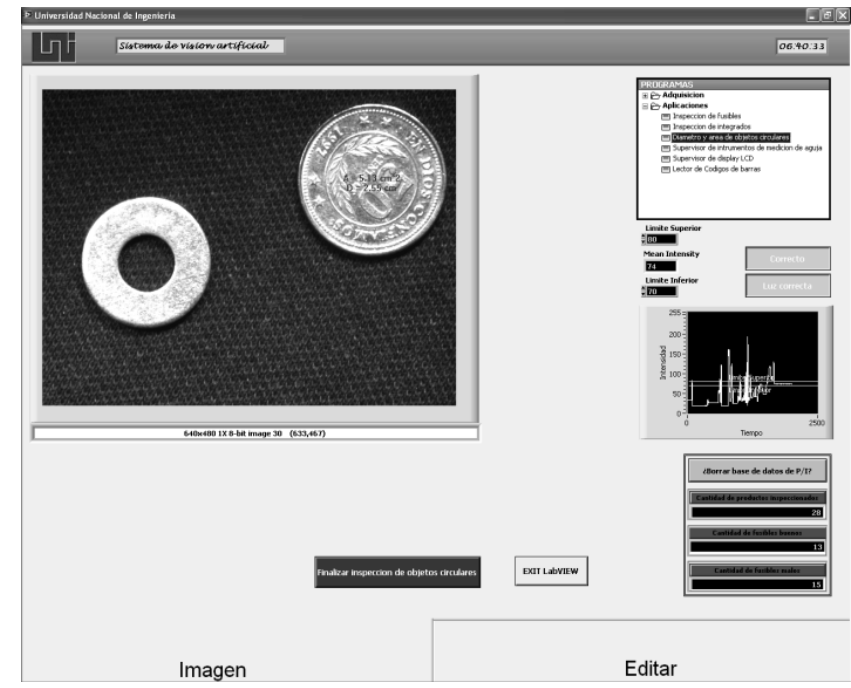

Fig. 5 Panel frontal para el Análisis de objetos circulares.

Los usos anteriores representan solamente algunas aplicaciones que los sistemas de visión artificial pueden tener en la automatización de los procesos. Dichas aplicaciones permiten a su vez, dar una idea de las bondades y alcances que puede llegar a tener este sistema en la industria.

\section{RESULTADOS Y DISCUSIÓN}

Se diseñó e implementó un sistema de visión artificial flexible que permitió realizar diversas tareas de inspección y procesamiento de imágenes en una gran variedad de objetos de producción industrial.

Este sistema fue probado a nivel de laboratorio con diferentes objetos para su análisis, y en todos los casos se obtuvieron resultados satisfactorios ya que al procesar las imágenes se obtuvo toda la información esperada de las variables de interés.

También se descubrió que es un sistema abierto a la investigación y al desarrollo de nuevas técnicas de procesamiento de imagen; ya que en el último programa se ilustran por separado algunas técnicas de reprocesamiento, como es el análisis espacial o de frecuencia (transformada de Fourier), etc. de una imagen.

Las capacidades del sistema pueden ser fácilmente ampliadas para el análisis molecular de objetos y para la detección de fallas en objetos de aluminio fundido, para lo cual se requeriría de una cámara que sea capaz de realizar estas tareas.

\section{CONCLUSIONES}

Fue llevado a cabo el diseño e implementación de un sistema de visión artificial teniendo como objetivo principal la enseñanza y adiestramiento de los estudiantes en el procesamiento y reconocimiento de imágenes para la automatización de procesos y control de calidad.

El sistema de visión está conformado por un conjunto de dispositivos, los cuales se listan a continuación:

- Cámara.

- Lente

- Tarjeta de adquisición

- Computadora

- Software

- Sistema de iluminación

- Estructura metálica.

Se implementaron diversas técnicas de procesamiento de imágenes para la realización de las aplicaciones del sistema de visión a través de la plataforma LabVIEW la cual es una herramienta muy poderosa en este campo.

Para optimizar el uso de una sola cámara se procedió a la construcción de una estructura mecánica que tiene como objetivo llevar a la cámara a cuatro posiciones distintas, lo que se logra a través del control automatizado de motores sincrónicos. El control de los motores se establece mediante la utilización de un PLC micrologic 1000. Además se diseñó una mesa giratoria en la que se posan los objetos a inspeccionar.

El sistema de iluminación que se implementó es a través de lámparas alógenas colocadas en distintas posiciones para lograr una iluminación uniforme, en dependencia del objeto a inspeccionar. La intensidad de luz de estas lámparas se logra a través de un dimmer el cual se controla con un motor paso a paso mediante el puerto paralelo de una computadora.

\section{AGRADECIMIENTOS}

Los autores agradecen a la Agencia Sueca de Cooperación Internacional para el Desarrollo (Asdi), por el apoyo financiero brindado para la realización de este trabajo. 


\section{BIBLIOGRAFÍA}

Informe de Auto evaluación de la Carrera de Ingeniería Electrónica 2005.

Manual de LabVIEW Machine Vision and Image Processing.

Restrepo, L. Una Introducción a la Visión de Máquina. (C) 1999-2007 http://www.infaimon.com

Web National Instruments, http://ni.com

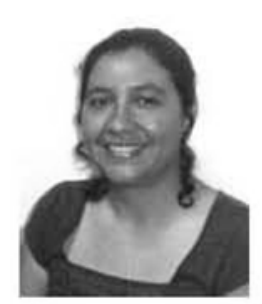

María Virginia Moncada, se graduó de Ingeniera Electrónica en la UNI en 1992. Realizó sus estudios de maestría en Industrial Measurements en Brunel University, London, 1995. Actualmente es profesora titular de la Facultad de Electrotecnia y Computación de la UNI. Ha trabajado en diferentes proyectos en el área de instrumentación y automatización industrial, con el auspicio del proyecto UNI-ASDI 